\title{
Techniques mathématiques utilisées en mécanique des fluides
}

\section{Mathematical techniques used in fluid mechanics}

\author{
PAR P. CASEAU, \\ ingÉNieur aU DÉPaRtement MÉGaniQue DEs Fluides,

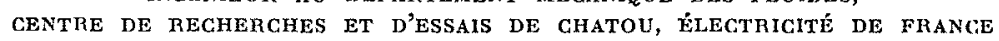

\begin{abstract}
Le but de cet exposé est de présenter -- sans suivre d'ailleurs un ordre logique très strict - quelques-uns des problèmes le plus fréquemment rencontrés en mécanique des fluides et quelques-unes des techniques adoptes pour les résoudre (calcul analogique - calcul numérique).

On a cherché à exposer les procédés usuels de résolution de ces problèmes et à discuter leurs chances de succès plutôt qu'à présenter de facon rigoureuse leurs fondements.
\end{abstract}

\begin{abstract}
A number of the more frequent problems of fluid mechanics and some of the techniques applied to them-i.e. analog and digital computation-are discussed. No particularly strict order of logic is followed; the aim is not to rigorously assess the reliability of the usual methods of approach to these problems, but rather to discuss the chances of their providing satisfactory answers.
\end{abstract}

\section{INTRODUCTION}

Nous allons d'abord situer les mathématiques par rapport aux autres moyens d'étude d'un problème donné. Il est en effet possible d'aborder un problème par trois voies :

\section{a) Etude expérimentale directe.}

\section{b) Etude expérimentale sur modèle.}

L'expérience à faire est en général schématisée (on néglige un certain nombre de paramètres), puis reproduite en similitude. C'est un fait général que la similitude repose sur une certaine simplification du problème. Les termes négligés, qui ne sont pas en similitude, donnent naissance à un «effet d'échelle».

\section{c) Etude théorique.}

L'expérience est alors schématisée d'une manière plus poussée que dans le cas précédent et on arrive à la construction d'un modèle mathématique. L'exploitation de ce modèle constitue la simulation de l'expérience initiale.

Les moyens d'exploitation d'un modèle mathématique sont de trois sortes:

- calcul analogique (réseaux, cuve électrolytique, calculateur analogique);

- calcul numérique (ordinateurs);

- résolution formelle, en général avec utilisation de tables numériques ou de fonctions «élémentaires».

Nous n'indiquons que pour mémoire les méthodes purement graphiques.

Le choix entre les méthodes $a$ ) $b$ ) ou $c$ ) ne peut se faire que selon chaque cas d'espèce. On peut dire (sans que cela soit absolument géné- 
ral) qu'une étude théorique est plus économique qu'un modèle, lui-mème plus économique qu'un prototype. Par contre, la part d'arbitraire et de schématisation est de plus en plus grande quand on passe de la première à la seconde, puis à la troisième méthode. Le manque de confiance de lutilisateur sera donc lui aussi, de plus en plus grand.

$$
* *
$$

Avant de pousser plus loin la discussion des méthodes mathématiques, nous allons examiner une classification des problèmes dont nous n'avons pas tenu compte jusqu'ici, et qui va préciser les conditions d'utilisation de chaque méthode. II nous faut en eflet distinguer :

\section{a) Les problèmes généraux.}

Les hypothèses envisagées varient dans un domaine large. On cherche donc :

- à construire des solutions;

- à classer les types de solutions, leurs conditions générales d'existence et leurs caractères essentiels.

On utilisera donc des modèles mathématiques simples.

A titre d'exemple, citons l'étude générale des divers générateurs d'électricité possibles en M.H.D.

\section{b) Les problèmes ( de Bureau d'étude ».}

Les hypothèses varient dans un domaine restreint. On cherche :

- les caractéristiques numériques de chaque solution;

- leur dépendance par rapport aux hypothèses.

Très souvent on y ajoutera une recherche d'optimum.

Sauf exception, on peut dire qu'on est ici dans le domaine d'élection du modèle réduit ou du calcul analogique. Il sera facile de tester les différentes «variantes» de la solution. Comme, d'autre part, on cherche des résultats numériques significatifs, les modèles mathématiques sont nécessairement complexes.

\section{c) Les problèmes de caractéristiques bien déterminées.}

Les hypothèses sont fixées une fois pour toutes. On cherche :

- à caractériser le mieux possible la solution;

- à vérifier un certain nombre de résultats et de calculs théoriques;

--- c'est ici le domaine du calcul numérique et de l'étude directe.

Le calcul des réacteurs, les études d'écoule- ments souterrains pour la recherche ou le stockage de gaz naturel en sont des exemples.

Après cetle courte introduction, nous allons maintenant évoquer quelques unes des questions essentielles qui se posent à l'ingénieur et au mathématicien, et définir quelques notions classiques. Notre but est simplement de présenter, sans ordre logique strict, un certain nombre de difficultés parmi les plus fondamentales, un certain nombre des techniques adoptées pour les résoudre.

\section{I. - PROBLEMES DE REPRESENTATION}

Sous ce titre, nous réunissons toutes les questions relatives à la «présentation » des résultats d'un calcul. Si nous cherchons une fonction $f(x)$ par exemple, par quoi allons-nous la caractériser? Nous pouvons hésiter entre la représentation graphique continue (calcul analogique), l'ensemble discret des valeurs en $x$ points (numérique ou réseau analogique) ou les coefficients d'un développement en série (numérique). Le choix entre les divers modes est lié aux impératifs de la méthode de résolution, mais aussi (et surtout) aux renseignements que nous désirons connaître. Il est rare en effet que l'on ait besoin de $f(x)$ en tout point. On cherchera plutôt une fonctionnelle liée à $f$. Par exemple :

- dans les problèmes de résonnance, de flambement, etc., on cherche la plus grande valeur propre d'un opérateur. C'est un nombre, mais c'est aussi :

$$
\lambda_{\max }==\max \frac{(f, \mathrm{~A} f)}{(f, f)}
$$

Il n'est donc pas interdit de penser que lon pourra obtenir par calcul analogique la fonction propre correspondante, puis intégrer numériquement pour avoir plus de précision sur $\lambda_{\max }$;

-- dans les problèmes d'hydrodynamique, le système des efforts sur un obstacle s'cxprime par :

$$
\vec{F}=\oint_{\Sigma}\left(\vec{u} \otimes \vec{u}-\frac{\overrightarrow{u^{2}}}{2}\right) \vec{n} d \sigma
$$

( $\Sigma)$ étant une surface quelconque entourant l'obstacle.

On tombe là encore sur une fonctionnelle quadratique. Il n'est peut-être pas nécessaire d'avoir une précision «locale» très grande.

$A$ ces problèmes, se rattache la notion de critère d'ćcart des solutions que nous allons obtenir. Qu'est-ce qui va décider qu'ume solution obtenue est bonne? Supposant la solution vraie connue, on peut caractériser par un seul nom- 
bre l'écart des deux solutions. On peut ainsi définir :

$f$ solution approchée,

$$
\varepsilon_{1}=\max |f-g|
$$

$g$ solution vraie.

ou encore, si les dérivées interviennent:

$$
\varepsilon_{2}=\max |f-g|+\max \left|f^{\prime}-g^{\prime}\right|
$$

Mais on peut définir des critères moins stricts :

$$
\varepsilon_{3}=\rho \mid f-g^{2}
$$

ou :

$$
\varepsilon_{4}=\int|f-g|^{2}+\int \mid f^{\prime}-g^{\prime 2}
$$

Ce deuxième type d'écart est plus souple. Il permet néanmoins d'obtenir des bornes d'erreur pour les fonctionnelles linéaires ou quadratiques liées à la solution. Il convient, en particulier, pour juger de la précision du calcul analogique.

Le choix de la représentation et du critère d'écart peut avoir une très grande importance. II en est ainsi en particulier pour les problèmes «mal posés». Pour ce type de problème, des conditions aux limites en apparence voisines peuvent engendrer des solutions différentes. Nous allons en donner un exemple très simple.

Soit donc une fonction $f(x, y)$ harmonique, périodique en $x$ (de période $2 \pi$ ). Nous supposons que l'on connait $f(x, 0)$ et $\partial f / \partial y(x, 0)$. Il s'agit, par exemple, d'un problème de conduction où l'on impose le flux $\partial f / \partial y(x, 0)$ et où l'on mesure la température $f(x, 0)$. Peut-on en déduire $f$ partout?

On verra facilement que pour $f$ quelconque, il n'en est rien, mais que l'on peut approcher $f$ par des fonctions $f_{n}$ pour lesquelles cela est possible. On voit ici l'importance du choix du critère d'écart. Si l'on choisit un écart quadratique (espace de Hilbert) on obtient un problème d'approximation quadratique classique. Si l'on choisit l'écart, on obtient le problème, plus général, de la recherche de la «meilleure solution» d'une équation dans un espace de Banach.

On consultera à ce sujet [2] où sont démontrés les résultats fondamentaux relatifs à ces problèmes.

\section{II. - PROBLEMES A UNE DIMENSION}

Ce type de problèmes est assez rare en mécanique des milieux continus (par opposition aux équations aux dérivées partielles qui sont la règle). Il est par contre très fréquent dès que l'on fait intervenir une schématisation suffisante des phénomènes (hydraulique, résistance des matériaux, etc.). On étudiera alors l'évolution, au cours du temps, d'un système à un nombre fini, mais grand, de parametres. On aboutira donc à des équations différentielles ou à des équations de convolution. Précisons d'abord une classification de ces problèmes :

\section{Problèmes de conditions initiales (conditions de Cauchy).}

Le traitement sera numérique ou analogique selon que l'on recherche la précision ou la rapidité.

\section{Problèmes de conditions aux limites (conditions} de Sturm-Lionville).

Le traitement sera essentiellement numérique, car on recherche avec précision les valeurs propres qui conduisent à des singularités (résonnances, flambement, etc.).

\section{Problèmes asymptotiques.}

On étudiera l'évolution finale d'un système (avec second membre) indépendamment des conditions initiales. C'est ici le domaine de la simulation ou du calcul analogique.

Il faut insister sur le fait que pratiquement tous les problèmes (à une dimension) de mécanique des fluides (ou dérivés de systèmes où intervient la mécanique des fluides) sont solubles par simulation et calcul analogique.

Qu'il s'agisse de cheminées d'équilibre, de réseaux hydrauliques, ou d'installations thermiques, etc., on peut considérer que tous ces systèmes ont leur équivalent analogique et que les méthodes d'étude, aussi bien théoriques que pratiques, sont à chercher de ce côté.

Avant d'ahandonner ce sujet somme toute bien connu, nous allons dire quelques mots d'une méthode d'approche classique des problèmes linéaires et des relations entre cette méthode et les moyens de calcul.

\section{Transformation de Fourier et filtres linéaires.}

Lorsque les problèmes à une diniension sont linéaires et homogènes par rapport au temps, ils s'expriment au moyen d'opérateurs de convolution : ce sont des filtres linéaires.

Considérons donc le problème type :

$$
\mathrm{K}_{*} f=g
$$

$\mathrm{K}$ : opération de convolution; 
$f$ : fonction inconnue,

$g$ : fonction donnée.

Si nous supposons que :

$$
f(t)=\int e^{i \sigma t} d \Phi(\sigma) \quad g(t)=\int e^{i a t} d \Gamma(\sigma)
$$

Nous pourrons en général introduire une fonction $\mathrm{K}(\sigma)$ et nous aurons :

$$
d \Gamma(\sigma)=\mathrm{K}(\sigma) \cdot d \Phi(\sigma)
$$

L'intérêt de la transformation de Fourier est donc, en changeant la représentation de la fonction, d'aboutir à une équation algébrique très simple. Résolvant l'équation sous la forme :

$$
d \Phi(\sigma)=\alpha(\sigma) d \Gamma(\sigma) \quad \alpha(\sigma)=\frac{1}{\mathrm{~K}(\sigma)}
$$

on pourra, sous certaines conditions, écrire :

$$
f=\mathbf{A}_{*} g .
$$

A sera alors la «réponse impulsionnelle» du système.

L'intérêt de ce genre d'analyse est triple :

- d'une part, il amène à changer de représentation. Dans le cas très fréquent où $f$ et $g$ sont des variables aléatoires stationnaires, la représentation de Fourier présente un très grand intérêt et l'analyse faite reste valable sans changement. D'ailleurs les physiciens utilisent très naturellement ce mode de présentation;

-. d'autre part, il ramène l'étude du système à l'étude de la convolution $A$, étude que l'on peut faire très facilement par voie analogique, de même que l'on peut très facilement trouver $K(\sigma)$ la «fonction de transfert» du filtre;

- enfin, il ne faut pas oublier qu'un grand nombre d'opérations faites théoriquement dans l'espace des phases ont leur correspondant dans l'espace de départ sous la forme d'une convolution qui se prête bien au calcul numérique :

Si :

$$
\begin{gathered}
d \Phi(\sigma)=\mathrm{A}(\sigma) d \mathrm{\Gamma}(\sigma) \\
f=\mathrm{A}_{*} g .
\end{gathered}
$$

Et si :

$$
\begin{gathered}
\mathrm{A}(\sigma) \simeq \Sigma \mathrm{A}_{n} e^{i \sigma \tau_{n}} \\
f \simeq \Sigma \mathrm{A}_{n} g\left(t+\tau_{n}\right)
\end{gathered}
$$

L'approximation sur l'opérateur de convolution A. doit toujours être faite en tenant compte de l'approximation correspondante dans l'espace des phases [3].

Nous allons donner un exemple d'application de ces remarques.

\section{Restitution DE LA hovle:}

Soit une houle cylindrique représentée sous la forme :

$$
h(x)=\int_{-\infty}^{\infty} e^{i k a} d Y(k)
$$

A la profondeur $z$, nous aurons :

$$
h(x, z)=\int_{-\infty}^{\infty} e^{i k x x} d Y_{1}(k)
$$

avec :

$$
d \mathrm{Y}_{1}(k)=e^{-|k| z} d \mathrm{Y}(k)
$$

ou :

$$
d \mathbf{Y}(k)=e^{+j k \mid z} d \mathbf{Y}_{1}(k)
$$

Si nous définissons :

D’où :

$$
\begin{gathered}
k(x)=\int_{-\infty}^{\infty} e^{i k x} e^{-|k| z} d k \\
k(x)=\frac{2 z}{x^{2}+z^{2}}
\end{gathered}
$$

$$
h(x, z)=\frac{1}{\pi} \int_{-\infty}^{\infty} \frac{z h(u) d u}{z^{2}+(x-u)^{2}},
$$

formule d'ailleurs tout à fait classique. Par contre :

$A^{*}(x)=\int_{-\infty}^{\infty} e^{i k x} e^{+|k| z} d k$ n'est pas convergente

Mais comme nous n'étudions qu'une houle dont les fréquences sont bornées, nous pouvons poser :

$$
\left.\mathrm{A}_{\mathrm{A}}\right|_{\text {yrox }}=\left.\int_{-k_{0}}^{k_{0}} e^{i k x z} e\right|^{k \mid z} d k
$$

ct nous aurons :

$$
\mathrm{A}_{a \text { lprox }}=\frac{1}{\pi\left(x^{2}+z^{2}\right)}\left[e^{k_{0} z}\left(z \cos k_{0} x+x \sin k_{0} x\right)-z\right]
$$

Nous avons approché l'opérateur $\left.e\right|^{k \mid z}$ par l'opérateur tronqué :

$$
\left.e\right|^{\mid k} \mid=\text { pour }|\mathbf{k}| \leqslant k_{0} \quad \text { et } \quad 0 \text { pour }|k| \geqslant k_{0}
$$

La formule ainsi obtenue est bonne pour une houle dont les fréquences sont inférieures à :

$$
\mathrm{N}_{0}=\frac{k_{0} c_{0}}{2 \pi}=\frac{\sqrt{k_{0} g}}{2 \pi}
$$


De la même façon, on peut montrer qu'il est possible de passer de $h(t, z)$ à $h(t, 0)$. L'opération ainsi introduite est, de même que (2), en général divergente. Mais il est possible d'on trouver des approximations convergentes valables pour des houles dont les fréquences sont limitées à une bande $\left(\mathrm{N}_{1}, \mathrm{~N}_{2}\right)$. Ceci réhabilite un peu la « restitution » de la houle point par point.

\section{III. - PROBLĖMES LINÉAIRES DANS LA THEORIE DES EQUATIONS AUX DÉRIVÉES PARTIELLES}

Il est remarquable de constater qu'arant l'utilisation extensive du calcul numérique, les seuls problèmes abordés par les mathématiques appliquées étaient les problèmes linéaires. Bien qu'ils aient maintenant perdu ce monopole, ils restent extrêmement importants.

\section{Linéarisation au voisinage d'un problème.}

Sommairement définie, la linéarisation est l'étude des perturbations au voisinage d'un écoulement connu. Nous allons indiquer quelques types de linéarisation.

Soit l'équation de Navier :

$$
\frac{\mathrm{D} \overrightarrow{\mathrm{U}}}{D t}=-\operatorname{grad} \mathrm{P}+\nu \nabla^{2} \overrightarrow{\mathrm{U}}
$$

et l'équation de Helmotz qui s'en déduit :

$$
\frac{\mathrm{D} \vec{\Omega}}{\mathrm{D} t}-\mathrm{Grad} \overrightarrow{\overline{\mathrm{U}}} \cdot \vec{\Omega}=\nu \nabla^{2} \vec{\Omega}
$$

Considérons un écoulement $\vec{U}_{0}$ vérifiant l'équation (3) et considérons un écoulement $\vec{U}$ voisin. Posons :

$\overrightarrow{\mathrm{U}}=\overrightarrow{\mathrm{U}}_{0}+\vec{u} \quad \vec{\Omega}=\vec{\Omega}_{0}+\vec{\omega} \quad|| \vec{u}|| / \mid \overrightarrow{\mathrm{U}}_{0} \| \ll 1$

et définissons :

$$
\frac{\mathrm{D} \overrightarrow{\mathrm{X}}}{\mathrm{D} t}=\frac{\partial}{\partial t} \overrightarrow{\mathrm{X}}+\mathrm{Gra \overline {d }} \overrightarrow{\mathrm{X}} \cdot \overrightarrow{\mathrm{U}}_{0}
$$

Nous obtenons, en ne retenant que les termes du premier ordre dans l'équation (4) :

$$
\begin{array}{r}
\frac{\overrightarrow{\mathrm{\omega} \omega}}{\mathrm{D} t}+\mathrm{Grad} \vec{\Omega}_{0} \cdot \vec{u}-\operatorname{Gra} \overline{\bar{a}} \vec{u} \cdot \vec{\Omega}_{0}-\operatorname{Gra\overline {a}} U_{0} \cdot \vec{\omega} \\
=\nu \nabla^{2} \vec{\omega}
\end{array}
$$

Si l'écoulement est à deux dimensions :

$$
\begin{aligned}
& \vec{u}=\vec{k} \wedge \operatorname{grad} \varphi(x, y) \text { Grad } \mathrm{U} \vec{\Omega}=0 \vec{\omega}=\nabla^{2} \varphi \vec{k} \\
& \overrightarrow{\mathrm{U}}_{0}=\vec{k} \wedge \operatorname{grad} \varphi_{0}(x, y) \vec{\Omega}_{0}=\nabla^{2} \varphi_{0} \vec{k}
\end{aligned}
$$

On arrive done a :

$$
\begin{aligned}
\frac{\mathrm{D}}{\mathrm{D} t}\left(\nabla^{2} \varphi\right)+\operatorname{gra\overline {d}}\left(\nabla^{2} \varphi_{0}\right)(\vec{k} \wedge & \operatorname{grad} \varphi) \\
& =\nu \nabla^{2} \nabla^{2} \varphi
\end{aligned}
$$

cette équation est l'équation d'Orr-Sommerfeld. Si nous supposons :

$$
\overrightarrow{\mathrm{U}}_{0}=\text { Cte } \quad \vec{\Omega}_{0}=0 \quad \text { Gräd } \mathrm{U}_{0}=0,
$$

nous obtenons :

$$
\frac{\mathrm{D} \omega}{\mathrm{D} t}=\nu \nabla^{2} \vec{\omega}
$$

c'esti la méthode proposée par Oseen.

Enfin, si nous admettons que $\overrightarrow{\mathrm{U}}_{0}=0$ nous obtenons :

$$
\frac{\partial \vec{s}}{\partial t}=\nu \nabla \overrightarrow{2}_{\omega,}
$$

c'est la méthode proposée par Stokes.

Il serait facile de montrer que ces méthodes de linéarisation s'étendent aux problèmes compressibles supersoniques ou subsoniques (théoric de Prandtl-Glauert par exemple) ainsi qu'aux problèmes à surface libre. Il s'agit donc d'une méthode générale d'étude des équations aux dérivées partielles de la Mécanique des fluides.

Nous allons maintenant préciser quelques notions qui sont fondamentales dans l'étude des problèmes linéaires.

\section{Problèmes auto-ad joints.}

Nous allons définir cette notion sur un exemple :

Soit l'écoulement d'un liquide conducteur soumis à un champ magnétique $\vec{B}_{0}$ constant. Moyennant certaines approximations [1], les équations électriques décrivant le phénomène sont:

$\left\{\begin{array}{c}\vec{j}=\sigma\left[\vec{e}+\vec{u} \wedge \vec{B}_{0}\right] \\ \operatorname{div} \vec{j}=0 \quad \operatorname{rot} \vec{e}=0\end{array}\right.$

$(\vec{j} \cdot \vec{n})=0 \quad$ aux frontières isolantes. 
Nous supposons, pour simplifier, l'écoulement fini et entièrement entouré par des parois non conductrices.

Soient alors deux écoulements $\vec{u}$ et $\overrightarrow{u^{\prime}}$, et les champs $\vec{j}, \overrightarrow{j^{\prime}}, \vec{e}, \overrightarrow{e^{\prime}}$ correspondants. On peut montrer que, si (V) est un volume entièrement contenu dans l'écoulement, (S) la frontière de ce volume :

$$
\int_{V} \overrightarrow{j^{\prime}}\left(\vec{u} \wedge \vec{B}_{0}\right)-\int_{\mathrm{v}} \vec{j} \cdot\left(\overrightarrow{u^{\prime}} \wedge \vec{B}_{0}\right)=\oint_{\mathrm{s}}\left(\vec{j} \mathrm{~V}^{\prime}-\overrightarrow{j^{\prime}} \mathrm{V}\right) \cdot \overrightarrow{\mathrm{n}} d \sigma
$$

ce qui montre que, pour l'ensemble de l'écoulement :

$\int \overrightarrow{j^{\prime}}\left(\vec{u} \wedge \overrightarrow{\mathrm{B}}_{0}\right) d v-\int \vec{j} \cdot\left(\vec{u}^{\prime} \triangle \overrightarrow{\mathrm{B}}_{0}\right) d v=0$

oul encore :

$\int \overrightarrow{e^{\prime}}\left(\vec{u} \wedge \overrightarrow{\mathrm{B}}_{0}\right) \sigma d v-\int \vec{e} \cdot\left(\overrightarrow{u^{\prime}} \wedge \overrightarrow{\mathrm{B}}_{0}\right) \sigma d v=0$

Or, considérons la résolution de (8). Nous avons :

$$
\vec{e}=-\operatorname{grad} \mathrm{V}
$$

$\left\{\nabla^{2} \mathrm{~V}=\operatorname{div}\left(\vec{u} \wedge \overrightarrow{\mathrm{B}}_{0}\right) \quad \frac{\partial \mathrm{V}}{\partial n}-\left(\vec{u} \wedge \overrightarrow{\mathrm{B}}_{0}\right) \cdot \vec{n}=0\right.$

Ainsi :

$$
\mathrm{V}=\lambda\left(\operatorname{div} \vec{u} \wedge \overrightarrow{\mathrm{B}}_{0}\right)
$$

$\lambda$ étant un opérateur linéaire,

et de même :

$$
\vec{e}=\mathrm{L}\left(\vec{u} \wedge \overrightarrow{\mathrm{B}}_{0}\right)
$$

L étant aussi un opérateur linéaire.

Si nous définissons le produit scalaire de deux champs de vecteurs $\overrightarrow{\mathrm{X}}$ et $\overrightarrow{\mathrm{Y}}$ comme :

$$
(\overrightarrow{\mathrm{X}}, \overrightarrow{\mathrm{Y}})=\int \overrightarrow{\mathrm{X}} \cdot \overrightarrow{\mathrm{Y}}^{*} \sigma d v(1)
$$

nous trouvons que, d'après (11),

$$
\left[\mathrm{L}\left(\vec{u} \wedge \overrightarrow{\mathrm{B}}_{0}\right), \overrightarrow{u^{\prime}} \wedge \overrightarrow{\mathrm{B}}_{0}\right]=\left[\vec{u} \wedge \overrightarrow{\mathrm{B}}_{0}, \mathrm{~L}\left({\overrightarrow{u^{\prime}}}^{\prime} \wedge \overrightarrow{\mathrm{B}}_{0} \cdot\right]\right.
$$

soit :

$$
[\mathrm{L}(\overrightarrow{\mathrm{X}}), \overrightarrow{\mathrm{Y}}]=[\overrightarrow{\mathrm{X}}, \mathrm{L}(\overrightarrow{\mathrm{Y}})]
$$

\footnotetext{
(1) $\vec{Y}^{\star}$ désigne le vecteur complexe conjugué de $\vec{Y}$.
}

Les opérateurs pour lesquels on peut définir un produit scalaire, et qui vérifient, pour ce produit, l'égalité (12) sont appelés auto-adjoints.

Montrons une propriété essentielle de ces opérateurs; soit :

$$
\varphi=\int\left(j^{2} / 2 \sigma\right) d v
$$

Considérons tous les potentiels qui vérifient :

$$
\frac{\partial V_{A}}{\partial \vec{n}}=+\left(\vec{u} \wedge \vec{B}_{0}\right) \vec{n} d \sigma
$$

et posons :

$$
\vec{e}_{\mathrm{A}}=-\operatorname{grad} V_{\mathrm{A}} \quad \vec{j}_{\mathrm{A}}=\sigma\left(\overrightarrow{e_{\mathrm{A}}}+\overrightarrow{\mathrm{U}} \wedge \overrightarrow{\mathrm{B}}_{0}\right)
$$

mais : $\operatorname{div} \vec{j}_{\Lambda} \neq 0$

Cherchons le minimum de :

$$
\Phi\left(\mathrm{V}_{A}, \vec{j}_{A}, \vec{u}\right) \text { lorsque } \mathrm{V}_{\mathrm{A}} \text { varie }
$$

Nous obtenons :

$$
\begin{aligned}
d \Phi=\int \vec{j}_{\mathrm{A}} \cdot \frac{d}{\sigma}\left(\vec{j}_{\mathrm{A}}\right)=\int \vec{j}_{\mathrm{A}} \cdot d \vec{e}_{\mathrm{A}} \\
=-\oint \vec{j}_{\mathrm{A}} d \mathrm{~V}_{\mathrm{A}} \cdot \vec{n}+\int \operatorname{div} \vec{j}_{\mathrm{A}} \cdot d \mathrm{~V}_{\mathrm{A}}
\end{aligned}
$$

mais :

$$
\overrightarrow{j_{A}} \cdot \vec{n}=0
$$

d'où :

done :

$$
d \Phi=\int \operatorname{div} \vec{j}_{\mathrm{A}} d \mathrm{~V}_{\mathrm{A}},
$$

$$
d \Phi=0<\operatorname{div} \vec{j}_{\mathrm{A}}=0^{(2)}
$$

Ceci est une propriété générale. Tout problème linéaire auto-adjoint est équivalent à une recherche d'extremum. L'interprétation physique de cette recherche est souvent intéressante et sera érigée en «principe»par les ingénieurs.

\section{Forme intégrale équivalente d'un problème d'équation aux dérivées partielles.}

Reprenons notre exemple, et plus précisément l'équation (10).

(2) Il est facile de voir que l'on aurait pu considérer les champs $\overrightarrow{j_{\mathrm{B}}}$ à divergence nulle : $d \Phi\left(j_{\mathrm{B}}\right)=0$ est alors équivalent à $\left(\partial V_{\mathrm{B}} / \partial n\right)=\left(\vec{a} \Lambda \overrightarrow{\mathrm{B}}_{0}\right) \cdot \vec{n}$; voir à ce sujet [4]. 
Considérons [1] les sphères de rayon $\varepsilon \rightarrow 0$ entourant 2 points A et B et soit $\vec{j}^{*}$ le courant engendré, en l'absence de tout écoulement :

$$
\left(\overrightarrow{u^{*}}=0\right)
$$

par une source unité placée au point $A$ et un puits unité placé au point $B$. Nous démontrerons alors que :

$\mathrm{V}_{\mathrm{A}}-\mathrm{V}_{\mathrm{B}}=\int \overrightarrow{j^{*}}\left(\vec{u} \wedge \overrightarrow{\mathrm{B}}_{0}\right) d v=\int \overrightarrow{\sigma e^{*}}\left(\vec{u} \wedge \overrightarrow{\mathrm{B}}_{0}\right)$

Là encore, il s'agit d'un résultat général. A partir des égalités (12), on peut trouver des représentations intégrales complètes de la solution. Dans une telle représentation, le «noyau» qui est ici le champ $\overrightarrow{j^{*}}$ s'appelle «fonction de Green du problème».

On peut se demander quels renseignements nouveaux apporte la formule (13). En fait, il est fréquent, et c'est le cas ici, que la fonction de Green soit beaucoup plus facile à déterminer par similitude ou analogie que la solution du problème initial.

En effet, on est dispensé de simuler le «second membre » l'écoulement $\vec{u}_{0}$ et le champ $\overrightarrow{\mathrm{B}}_{0}$. La fonction $\overrightarrow{e^{*}}$ ne dépend que de la géométrie du problème et des points $A$ et $B$. On pourra ensuite exploiter numériquement la formule (13).

Dans la formule (13) le problème est complètement résolu. II peut arriver au contraire qu'il n'en soit pas ainsi et que l'on essaie de reconstituer la solution au moyen de singularités simples, ce qui conduira à des équations intégrales du type de Fredholm.

Celles-ci peuvent être de première ou de deuxième espèce. Ainsi la représentation de l'écoulement au voisinage d'un corps axisymétrique effilé, par un ensemble de doublets conduit à une équation de première espèce.

Ces équations ont eu, en général, mauvaise presse, car elles étaient singulières et difficiles à résoudre. En fait, il convient de s'inspirer des considérations sur l'approximation déjà évoquées et de chercher des solutions voisines de la solution cherchée, par exemple par combinaison linéaire. Il est à remarquer que c'est justement à propos de problèmes de potentiel «mal posés $»$ que les recherches sur l'approximation se sont développées. La recherche de solution par combinaison de singularité est certainement une technique fondamentale pour les problèmes linéarisés que nous évoquions au début de ce paragraphe.

\section{Problèmes paraboliques.}

Nous avons jusqu'ici abordé des problèmes du type :

$$
\mathrm{A} f=g
$$

A opérateur linéaire, $g$ fonction connue, $f$ inconnue.

Considérons maintenant l'équation :

$$
\frac{\partial f}{\partial t}-\mathrm{A} f=g
$$

on encore :

$$
\frac{\mathrm{D} f}{\partial t}-\mathrm{A} f=\frac{\partial f}{\partial t}+\mathrm{U} \frac{\partial f}{\partial x}-\mathrm{A} f=g
$$

Si nous supposons que $\mathrm{U}=\mathrm{U}(y, z) Y_{\mathrm{A}} \mathrm{A}=$ opérateur de $x$, notre problème sera homogène en $x$ et $t$. Restreignons l'étude aux problèmes homogènes et permanents :

$$
U \frac{\partial f}{\partial x}=A f
$$

Posons alors :

$$
f=e^{\lambda x}
$$

Nous aurons :

$$
\lambda \mathrm{U} f=\mathrm{A} f \quad \text { ou } \lambda f=\frac{\mathrm{A}}{\mathrm{U}} f
$$

Si l'opérateur A/U est auto-adjoint (et de plus complètement continu), nous aurons les théorèmes suivants :

- les valeurs de $\lambda$ admissibles forment une suite distincte de «valeurs propres » réelles;

- les fonctions propres associces sont orthogonales et forment, sous certaines conditions, un système complet.

La solution générale s'écrit done :

$$
\left\{\begin{aligned}
i & =\Sigma a_{n} e^{-\lambda_{n} x} g_{n}(y, t) \\
a_{n} & =\left(f, g_{n}\right)
\end{aligned}\right.
$$

Les problèmes physicques stables (du type diffusion) sont caractérisés de plus, par des opérateurs A définis négatifs, ce qui entraîne que les valeurs $\lambda_{n}$ sont négatives et que les perturbations décroissent comme $e^{-\lambda_{n^{*}} \text {, }}$

Les applications théoriques on pratiques de ces propriétés sont très nombreuses. C'est pourquoi la recherche des premières valeurs propres, qui mesurent l'atténuation de la quantité $f$ le long de l'écoulement, est un problème fondamental.

Insistons sur le fait que c'est un problème autonome, indépendant du caleul \&brutal » de 
l'évolution de $f$. Très souvent, les spécialistes du calcul numérique considèrent avec méfiance la formule (14) qui n'est pas, effectivement, une formule de calcul numérique (la résolution directe est souvent meilleure). Mais - et c'est ici que la collaboration entre ingénieurs et calculateurs prend tout son sens - très souvent la forme (14) est plus intéressante que le résultat numérique brut. Elle permet, pour l'étude de l'évolution asymptotique en particulier, de bien savoir ce qu'il faut mesurer ou calculer et d'utiliser le mode d'approche le plus approprié.

\section{IV. - RETOUR SUR LES METHODES NUMERIQUES}

Pour terminer, nous allons passer en revue les techniques numériques de résolution des équations aux dérivées partielles.

\section{Méthode classique - Discrétisation.}

On représente la fonction cherchée par ses valeurs numériques aux nœuds d'un réseau recouvrant le domaine de résolution. Le réseau est, en général, régulier. Restent dans cette catégorie : - les réseaux maillés analogiques (équation de la chaleur, ou équation de Laplace);

- les méthodes classiques de résolution sur ordinateur.

\section{Reprësentation par des singularités - Equa- tions intégrales.}

On reconstitue la fonction cherchée comme somme de solutions élémentaires. La considération des conditions aux limites conduit en général à une équation intégrale. Malheureusement, cette méthode ne s'applique qu'aux problèmes linéaires.

Bien qu'il ne soit pas impensable d'exploiter cette idée en calcul analogique, l'application se fait en général sur ordinateur. Les difficultés de résolution sont du même ordre qué pour la méthode $\mathrm{A}$.

\section{Techniques hybrides.}

Les calculateurs analogiques ètant organisés surtout pour résoudre les équations différentielles, il est tentant de se ramener à ce cas en rem- plaçant l'équation de départ par une équation différentielle aux différences. Il suffit de discrétiser par rapport à une seule des variables, en gardant l'autre continue. Mais pour que cette technique soit applicable, il faut que l'on puisse mettre en mémoire le résultat du calcul à chaque pas. En effet, on arrivera, par exemple, à une équation du type :

$$
\mathrm{L}_{x}\left(f_{n}\right)=\mathrm{D}_{x}\left(f_{n-1}\right)
$$

$L_{x}, \mathrm{D}_{x}$ opérateurs différentiels en $x$;

$f_{n}=f(x, y=n h) \quad f_{n-1}=f[x, y=(n-1) h]$.

Pour résoudre l'équation en $f_{n}$ il faut donc mettre en mémoire le résultat du calcul sur $f_{n-\ldots 1}$. Ce stockage se fait, jusqu'à présent, sous forme numérique : ce qui signifie que l'on a effectué, en fait, une discrétisation également en $x$, pour pouvoir stocker $f_{n}$. Un des avantages de la technique proposée disparaît donc.

\section{Méthode de Galerkine - Représentation par des séries de fonctions.}

Soit par exemple, l'équation :

$$
\frac{d f}{d t}=\mathrm{Af}
$$

On la remplacera par l'équation :

$$
\frac{d \mathrm{P}}{d t}=\mathrm{AP}
$$

où :

$$
\mathrm{P}=\Sigma x_{n} g_{n} \quad\left(g_{0}, g_{1} \ldots g_{\mathrm{N}}\right)
$$

suite de fonctions arbitraires.

On arrive donc à un système différentiel :

$$
\frac{d}{d t}\left[x_{n}\right]=\mathrm{A}\left[x_{n}\right]
$$

Malheureusement, il convient de signaler que : - les méthodes numériques d'intégration (Runge-Kutta) sont longues et coûteuses. Leur application au système (18) a toujours été plus lourde que la résolution directe par la méthode classique;

- les méthodes analogiques, pour le cas de système d'ordre élevé, ne sont pas encore suffisamment au point.

Néanmoins, il semble que dans un grand nombre de cas (par exemple celui que nous avons cité) on peut isoler et calculer numériquement quelques termes dominants de l'évolution de $f(x, y, t)$. La méthode de Galerkine serait pré- 
cieuse pour caractériser l'évolution du « reste». Un petit nombre de fonctions de base seraient alors nécessaires.

\section{Méthodes graphonumériques.}

Ce sont les méthodes classiques utilisant les caractéristiques, qui ne sont donc applicables qu'aux problèmes hyperboliques dont nous avons peu parlé. Leur défaut est d'être rapidement très complexes lorsque le nombre des dimensions augmente.

\section{BIBLIOGRAPHIE}

[1] P. Blanc-FÉraud. - Ecoulements fluides soumis a un champ magnetique. Application à la mesure des vitesses. Bulletin du C.R.E.C., supplément au $\mathrm{n}^{\circ}$, juillet 1962 .

[2] Lago. - Meilleure solution de $A u=g$. Revue française de traitement de l'information, $\mathrm{n}^{\circ} 1,1^{\mathrm{or}}$ trimestre 1963.

[3] Arsac. - Transformation de Fourier et theorie des distributions, Dunod, 1961.

[4] Covnant and Hilbent. - Methods of Mathematical Physics, vol. I.

\section{DIS CUS SION}

président : M. Grbrat

M. le Président remercie M. Cassau d'avoir̈ fait le [nul' des utilisations des mathomatiques par l'ingénieur et d'avoir mis l'accent sur ce qu'il $y$ avait à retirer, pour celui qui s'occupe surtout de mécanique des fluides, des techniques mathématiques.

M. le Président ouvre ensuite la discussion à propos des critères d'erreurs. Il rappelle que l'on peut se contenter de la condition très dure de la différence la plus grande existant entre deux points (expérience et fonction théorique), à condition que ceci corresponde exactement au sujet cherché, puis il mentionne l'intérêt de certains tests statistiques correspondant à des séries de problèmes, ces tests ayant pour but d'ajuster des résultats d'expérience à une fonction théorique. Peut-être les considérations développées par M. CASEAU pourraientelles être affinées à l'aide de tests statistiques?

M. le Président demande ensuite des précisions sur le problème évoqué par M. Caseau à propos de la conduction de la chaleur: d'après $M$. Caseau, si l'on pose le problème de facon simple et claire, apparemment bien définie, les difficultés rencontrées sont telles qu'il $n^{\prime} y$ a pas de solution. Si l'on se contente d'une certaine arpproximation, qu'on se fixe à l'avance, il y a une solution. Mais à quel moment les mathématiques interviennent-elles pour singulariser la solution? Et quel est le critère qui fait que, parmi une infinité de solutions, lune soit meilleure?

M. Caseau répond que, du point de vue général, il a montré que le critère quadratique permet d'obtenir des bornes d'erreurs sur certains résultats comme les intégrales, mais que, pour l'analyse d'une courbe entière, il taudrait certainement utiliser les critères mentionmés par M. le Président.

En ce qui concerne le problème de la conduction de la chaleur, M. Cassau rappelle que, si l'on développe la solution en série de Fourier, chaque harmonique se décompose en deux. Une partie tend vers 0 comme $e^{-n y}$, l'autre vers l'infini comme $e^{+n y}$. Pour que la solution ait un sens, il faut done que le développernent en série de $f$ et de $\partial f / \partial y$ ne soit pas quelconque. Mais, comme $f$ est le résultat d'une mesure, toute erreur sur f va engendrer des harmoniques divergents.

Pratiquement, si l'on tronque le développement jusqu'au degré $n$, et si l'on impose un critère d'écart valable, on peut avoir une assez bonne idée de ce qui va se passer. L'indétermination, toutefois, est assez semblable à celle qui entache la représentation d'une lonction par un polynome. A partir d'un certain ordre, l'information supplémentaire conduit à des résultats sans valeurs. On ne peut obtenir de résultats parfaitement satisfaisants. Il faut néammoins remarquer que, lrès souvent, trois ou quatre harmoniques suffisent à représenter convenablement le problème.

M. le Président ratfache ce problème à la théorie des séries divergentes et rappelle la méthode utilisćc on astronomie par Henri Poincare : celle-ci conduisait a une série divergente et la précision s'améliorait, avee la prise en compte des termes successifs, jusqu'a un certain point puis s'amenuisait. M. le Président demande à M. Caseau si, dans son article, il s'agit de séries divergentes.

M. Caseau répond que ce n'est pas exactement cela. Une des difficultés essentielles, que $M$. LAGo a eu à résoudre, était de montrer que, quel que soit le critère d'écart imposé, à condition que l'espace obtenu vérifie certaines propriétés, il $y$ a toujours une solution; la question de la convergence du phénoméne vers la solution réelle, a supposer qu'elle existe, est plus difficile à résoudre.

M. le Président remercie M. Cassay pour son exposé, qui fera benucoup réfléchir, peut-être même sur des domaines plus éloignés de notre activité que ceux de la mécanique des fluides. 